\title{
Characterization of asthma and risk factors for delayed SARS-CoV-2 clearance in adult COVID-19 inpatients in Daegu
}

\author{
Su-Jeong Kim${ }^{1}$, Chang-Gyu Jung ${ }^{2}$, Ji Yeon Lee ${ }^{2}$, Gunwoo Kim ${ }^{3}$, Hyun Jung Jin ${ }^{4}$, and \\ Han-Ki Park ${ }^{5}$ \\ ${ }^{1}$ Kyungpook National University Hospital \\ ${ }^{2}$ Keimyung University Dongsan Hospital \\ ${ }^{3}$ Daegu Fatima Hospital \\ ${ }^{4}$ Yeungnam University School of Medicine and College of Medicine Medical Library \\ ${ }^{5}$ Kyungpook National University School of Medicine
}

August 6, 2020

\section{To the Editor:}

The spread of the coronavirus disease-2019 (COVID-19) remains a worsening global health crisis. Although many studies have reported risk factors for severe COVID-19, asthma characterization in COVID-19 is still controversial, with different early reports from China and recent reports from the Europe and United States. ${ }^{1}$ Prolonged viral shedding is not only a risk factor for poor outcome of COVID-19, but also clues to host immune response against the virus. However, there is limited data on this except for results from relative small group studies. ${ }^{2}$ In this study, 2200 adult patients hospitalized for COVID-19 in Daegu were evaluated for prevalence of asthma and clinical outcomes with COVID-19 according to asthma. In addition, the risk factors for delayed viral clearance were evaluated.

The prevalence of asthma in patients with COVID-19 was 3.2\% which was not different from its prevalence in the Korea National Health and Nutrition Examination Survey (KNHANES) (Figure 1A and Table S1). By age group, the prevalence of asthma showed a similar U-shaped pattern as the general prevalence pattern in Korea. However, the prevalence of asthma in the 19-29-year age group (2.1\%) was lower than that of KNHANES (Figure 1B).

Table S2 compares the characteristics between the asthma group and the non-asthma group. Older age, overweight, and comorbidity of chronic obstructive pulmonary disease, and initial symptoms of dyspnea and nausea/vomiting were more common in the asthma group. Compared with the non-asthma group, the asthma group had a greater risk of death $(13.6 \%$ vs. 6.4\%, $P=0.02)$ and a greater need for high-flow oxygen therapy $(18.2 \%$ vs $.10 .5 \%, P=0.048)$ (Figure $1 \mathrm{C}$ and Table S3). The higher mortality rate in asthma patients compared with non-asthmatic patients was particularly noticeable in female and overweight patients. Older patients ( $>65$ years) with asthma tended to have a higher mortality rate than those without asthma (Figure 1D). After adjusting for potential confounders, asthma had no significant association with clinical outcomes of COVID-19 (Figure 1E and Table S4). Meanwhile, older age, male gender, and comorbid diseases including overweight, diabetes, chronic kidney disease, cancer, autoimmune disease, dementia, and other psychological disorder were significant risk factors for mortality (Tables S5 and S6).

Asthma is considered to have a lower risk of death than other well-known risk factors. ${ }^{3,4}$ However, asthma is a heterogeneous disease and is often associated with atopic and eosinophilic asthma in younger patients. 
Meanwhile, obese asthma and elderly asthma are known to have common neutrophilic phenotypes. ${ }^{5,6}$ The recent results of higher expression of COVID-19 receptors in respiratory specimens with neutrophilic asthma phenotype compared with the eosinophilic asthma phenotype. ${ }^{7}$ Considering prevalence and clinical outcome results, it is possible that neutrophilic asthma is a risk factor for infection and poor prognosis of COVID-19 rather than eosinophilic asthma.

When delayed viral clearance was divided into two groups based on 30 days, 906 patients were included in the non-delayed viral clearance group and 415 patients in the delayed viral clearance group. After adjusting for potential confounders, delayed viral clearance was not significantly associated with asthma (Figure 1E and Table S4). However, older age $>65$ years (Odds ratio (OR) 2.002, 95\% Confidence interval (CI) 1.292-3.101; $P=0.002$ ), comorbid diseases including dementia (OR 3.123, 95\% CI 1.833-5.321; $P<0.001$ ), and other psychological disorder (OR 2.084, 95\% CI 1.178-3.687; $P=0.012$ ), initial symptom of skin rash (OR 15.943, 95\% CI 1.613-157.535; $P=0.018)$, and initial laboratory abnormalities including hemoglobin $<10 \mathrm{~g} / \mathrm{dL}$ (OR 2.156, 95\% CI 1.161-4.003; $P=0.015)$ and C-reactive protein (CRP) [?]1.0 mg/dL (OR 1.588, 95\% CI 1.061-2.377; $P=0.025)$ were significant risk factors for delayed viral clearance. On the other hand, male sex (OR 0.752, 95\% CI 0.567-0.997; $P=0.047$ ), hypertension (OR 0.704, 95\% CI 0.519-0.953; $P=0.023$ ), and initial symptom of headache (OR $0.673,95 \%$ CI $0.485-0.932 ; P=0.017$ ) were significant protective factors for delayed viral clearance (Figure 2A and Table S7). In particular, when limited to the mild COVID-19 group classified as no activity limitations in the outcome parameters, older age, dementia, initial symptoms of skin rash and headache, and initial hemoglobin $<10 \mathrm{~g} / \mathrm{dL}$ showed significant differences (Figure 2B, Table S8).

Several factors related to the nervous system were identified as important risk factors for delayed viral clearance. Previous studies have shown that the coronavirus can initially invade the peripheral nerves and enter the central nervous system through a synapse path. ${ }^{8}$ It is hypothesized that the ability of the immune system to find and remove viruses that have penetrated the nervous system is important for virus clearance. Male sex, hypertension and elevated CRP did not show a significant difference when analyzed only mild patients, and these may be indicators associated with severity rather than a direct effect on viral clearance.

Anti-inflammatory drugs such as hydroxychloroquine and systemic steroid were shown to be risk factors for mortality and delayed viral clearance (Table S6 and S8). These medications were used more often when the hospitalization period was extended or when showing poor prognosis factors. Notwithstanding these, our results suggest that anti-inflammatory drugs need to be used with proper consideration of appropriate indications.

On May 9, 2020, there were 6,859 patients with PCR-confirmed COVID-19 in Daegu. This data excluded asymptomatic or minimal symptomatic patients who did not require hospitalization. However, our study covered almost all hospitalized patients diagnosed with COVID-19 in Daegu from February to May ,therefore, selection bias is minimized. ${ }^{9}$ In Korea, most hospitals decided to terminate the quarantine by repeating PCR every week. In addition, the Korea Centers for Disease Control \& Prevention (KCDC) thoroughly managed the criteria for quarantine termination and PCR results. Through this, in our study, we were able to perform a large-scale study to confirm the risk factors for delayed viral clearance.

In summary, despite the positivity of differences depending on phenotypes, the prevalence of asthma was not significantly different in patients with COVID-19, and asthma did not affect the outcomes of COVID-19. Age, dementia, and initial presentations of headache, skin rash, and anemia were independently associated with viral clearance.

\section{Reference}

1. Johnston SL. Asthma and COVID-19: is asthma a risk factor for severe outcomes? Allergy 2020; 75: $1543-5$.

2. Yan D, Liu X-Y, Zhu Y-n, et al. Factors associated with prolonged viral shedding and impact of Lopinavir/Ritonavir treatment in hospitalised non-critically ill patients with SARS-CoV-2 infection.Eur 
Respir J 2020; 56: 2000799

3. Zhou F, Yu T, Du R, et al. Clinical course and risk factors for mortality of adult inpatients with COVID-19 in Wuhan, China: a retrospective cohort study. Lancet 2020; 395: 1054-62.

4. Docherty AB, Harrison EM, Green CA, et al. Features of 20133 UK patients in hospital with covid-19 using the ISARIC WHO Clinical Characterisation Protocol: prospective observational cohort study. $B M J$ 2020 ;369: m1985.

5. Rufo J, Taborda-Barata L, Lourenço O. Serum biomarkers in elderly asthma. J Asthma 2013; 50(10): 1011-9.

6. Telenga E, Tideman S, Kerstjens H, et al. Obesity in asthma: more neutrophilic inflammation as a possible explanation for a reduced treatment response. Allergy 2012; 67(8): 1060-8.

7. Bradding P, Richardson M, Hinks TS, et al. ACE2, TMPRSS2 AND FURIN GENE EXPRESSION IN THE AIRWAYS OF PEOPLE WITH ASTHMA-IMPLICATIONS FOR COVID-19. J Allergy Clin Immunol 2020; 146: 208-11.

8. Vellingiri B, Jayaramayya K, Iyer M, et al. COVID-19: A promising cure for the global panic. Sci Total Environ 2020; 725: 138277.

9. Kim J-H, An JA-R, Min P-k, et al. How South Korea responded to the Covid-19 outbreak in Daegu. NEJM Catalyst Innovations in Care Delivery . 2020; 1(4).

Sujeong Kim, MD, ${ }^{1 *}$ Chang-Gyu Jung, MD,${ }^{2 *} \mathrm{Ji}$ Yeon Lee, MD,${ }^{3}$ Gunwoo Kim, MD, PhD,${ }^{4}$ Hyun Jung Jin, $\mathrm{MD}, \mathrm{PhD},{ }^{5+}$ Han-Ki Park, MD,${ }^{6+}$

${ }^{1}$ Department of Allergy and Clinical Immunology, School of Medicine, Kyungpook National University, Kyungpook National University Hospital, Daegu, Korea

${ }^{2}$ Division of Allergy and Clinical Immunology, Department of Internal Medicine, Keimyung University Dongsan Hospital, Daegu, Korea

${ }^{3}$ Divison of Infectious Disease, Department of Internal Medicine, Keimyung University Dongsan Hospital, Daegu, Korea

${ }^{4}$ Department of Allergy and Rheumatology, Fatima Hospital, Daegu, Korea

${ }^{5}$ Department of internal medicine, Medical school of Yeungnam University, Daegu, Korea

${ }^{6}$ Department of Allergy and Clinical Immunology, School of Medicine, Kyungpook National University, Kyungpook National University Chilgok Hospital, Daegu, Korea

*: These authors contributed equally to this work

${ }^{+}$: Correspondence to the following authors

Professor Han-Ki Park, MD

Department of Allergy and Clinical Immunology, School of Medicine, Kyungpook National University Chilgok hospital, Hoguk-ro, Buk-gu, Daegu 41404, Republic of Korea

Tel: $+82-53-200-2617$

Fax: +82-53-200-2029

Email: haan33@gmail.com

Professor Hyun Jung Jin, MD, PhD

Department of internal medicine, Medical school of Yeungnam University, 170 Hyeonchung-ro, Nam-gu, Daegu 42415, Korea. 
Tel: $+82-53-620-3830$

Fax: $+82-53-620-3849$

Email: jhj0619@yu.ac.kr

Conflicts of Interest

The authors declare no competing financial interest.

\section{Acknowledgments}

This work was supported by the Research Program of Medicity Daegu Council funded by Daegu Metropolitan City (fund code COVID19_DM05)

\section{Figure legends}

Figure 1. Characterization of asthma in adult COVID-19 inpatients. (A) Prevalence of asthma in COVID-19 and in the KNHANES. (B) Prevalence by age group in COVID-19 and in the KNHANES. (C) Univariate analysis of clinical outcomes according to asthma. (D) Death and ongoing care according to the asthma subgroups. (E) Forest plot showing odds ratios (ORs) of asthma for the clinical outcomes. ORs are adjusted for age, sex, body mass index, smoking history, underlying comorbidity, and treatment for COVID-19 by multivariate logistic regression model.

Abbreviations: KNHANES $=$ the Korea National Health and Nutrition Examination Survey; COVID-19= coronavirus disease-2019; ICU = intensive care unit; $\mathrm{BMI}=$ body mass index; PCR = polymerase chain reaction; SARS-CoV-2 $=$ severe acute respiratory syndrome coronavirus 2 .

Figure 2. Forest plot showing odds ratios (ORs) for risk factors for delayed viral clearance ( $>30$ days). (A) Total patients $(\mathrm{N}=1321$; the delayed viral clearance group $=415)$. (B) Mild (no activity limitation) group $(\mathrm{N}=938$; the delayed viral clearance group $=281)$. ORs are adjusted for age, sex, body mass index, smoking history, underlying comorbidity, and medication for COVID-19 by multivariate logistic regression model.

Abbreviations: COVID-19 = coronavirus disease 2019; $\mathrm{Hb}=$ Hemoglobin; CRP $=$ C-reactive protein. 
(A)

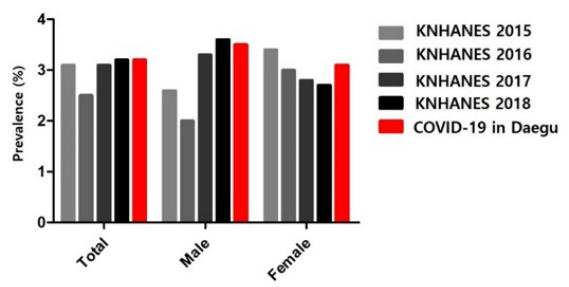

(B)

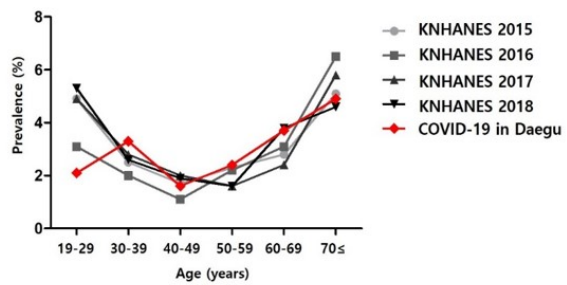

(C)

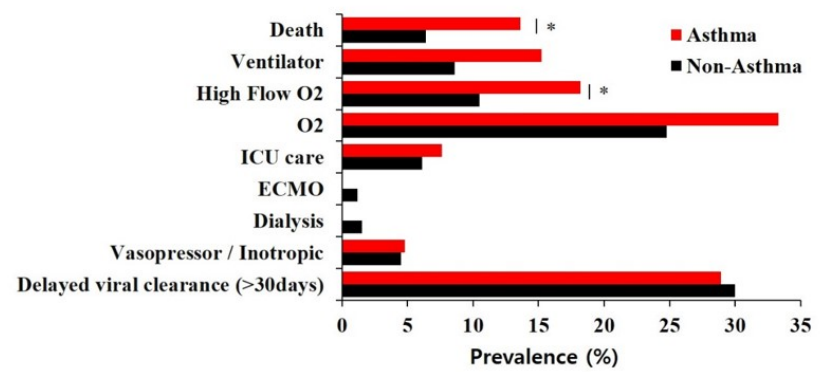

(D)

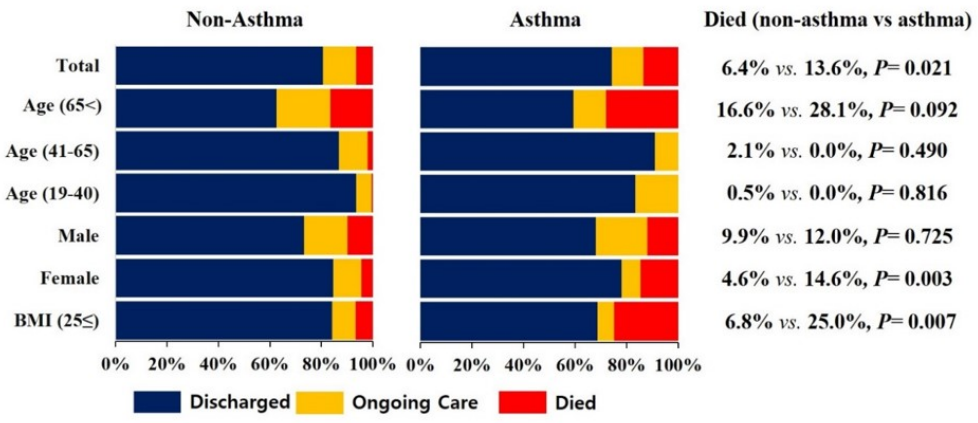

(E)

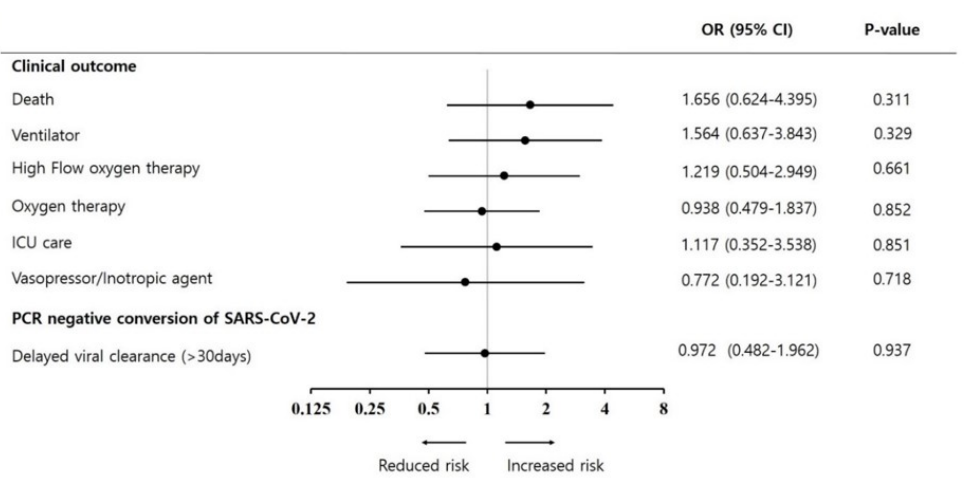


(A)

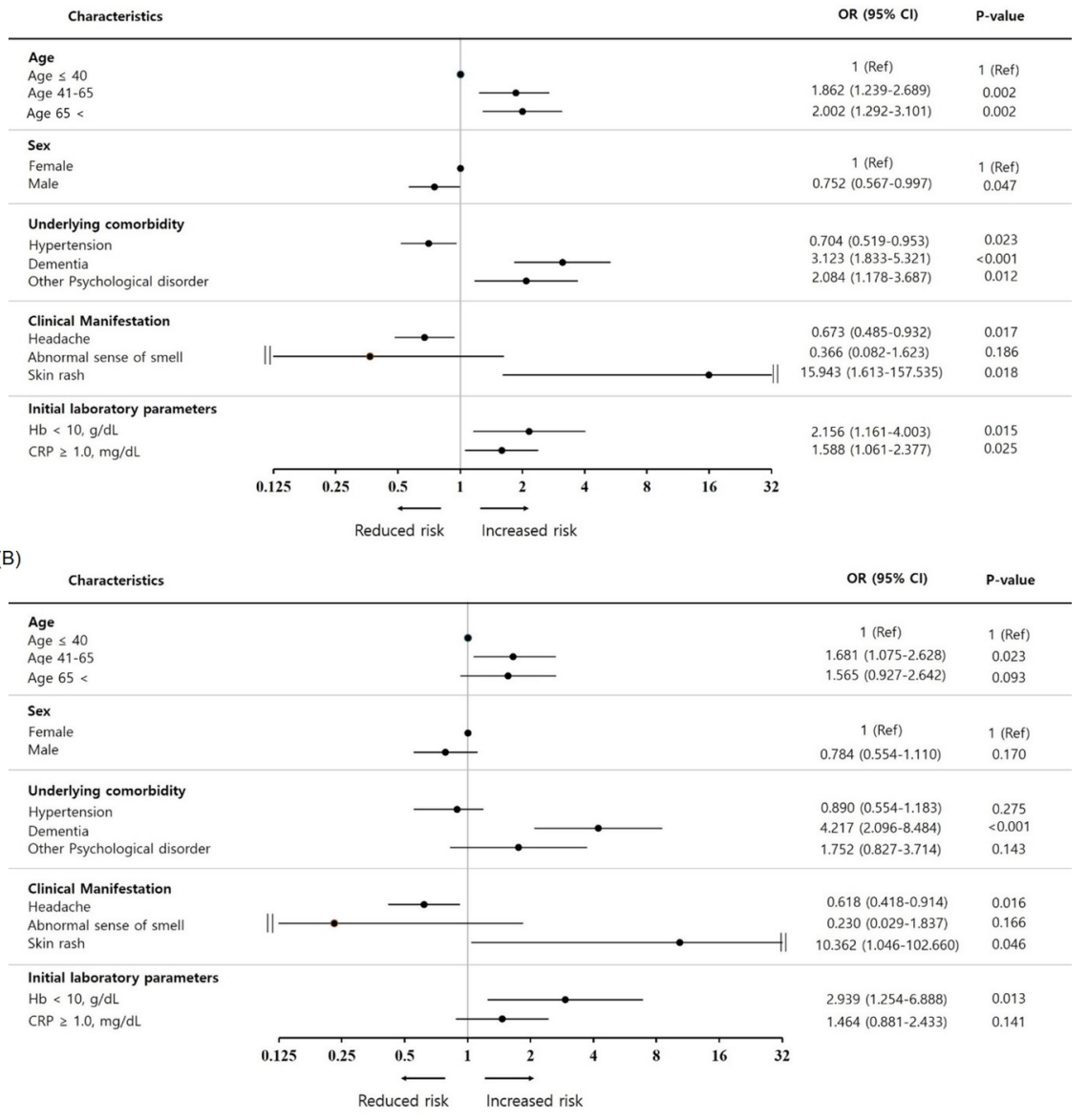

